\title{
Se mouvoir au sein du monde de l'information : comment les personnes parlent de leur activité d'information
}

Moving within the Information World: How People Report Their Information-

Related Activities

\section{Brigitte Guyot}

\section{OpenEdition}

Journals

Édition électronique

URL : http://journals.openedition.org/edc/1052

DOI : 10.4000/edc. 1052

ISSN : 2101-0366

\section{Éditeur}

Université Lille-3

\section{Édition imprimée}

Date de publication : 1 décembre 2009

Pagination : 101-118

ISSN : 1270-6841

Référence électronique

Brigitte Guyot, «Se mouvoir au sein du monde de l'information : comment les personnes parlent de

leur activité d'information », Études de communication [En ligne], 33 | 2009, mis en ligne le 01 décembre 2011, consulté le 19 avril 2019. URL : http://journals.openedition.org/edc/1052 ; DOI : 10.4000/ edc. 1052

Ce document a été généré automatiquement le 19 avril 2019

(c) Tous droits réservés 


\title{
Se mouvoir au sein du monde de l'information : comment les personnes parlent de leur activité d'information
}

\author{
Moving within the Information World: How People Report Their Information- \\ Related Activities
}

Brigitte Guyot

1 A l'heure où nombre de discours dans la société irriguent la vie d'une entreprise, certains se matérialisent en autant de dispositifs de communication, notamment empruntés au web 2.0 mettant en avant les échanges, le partage, les réseaux sociaux, la publicisation de soi. Nous souhaiterions porter le regard sur les façons très concrètes dont les acteurs mobilisent de l'information et créent de nouvelles formes d'interaction avec elle. Ces nouvelles prescriptions viennent prolonger une longue diversification d'outils et de systèmes d'information et mettent l'utilisateur dans des positions de dépendance, d'autonomie ou de liberté, selon l'angle d'analyse sous lequel on se place. Nous voudrions vérifier les écarts entre les initiatives prises pour améliorer la gestion ou l'accès à l'information et le vécu des acteurs, étudier comment ils s'ajustent à cette multiplication de systèmes d'information, dans lesquels ils entrent et sortent. C'est mesurer ces coûts cachés dont fait partie l'information, difficiles à mesurer quand la productivité reste le critère déterminant mais qui occupent une large part du temps de travail.

2 Ce point d'entrée par le poste de travail donne à voir un enrôlement de dispositifs et d'outils; l'idée n'est pas ici d'étudier l'interaction homme-machine mais les façons dont chacun produit et utilise de l'information, pour en relever les difficultés ainsi que la relative solitude dans laquelle il se trouve pour connaître les sources potentiellement utiles ou tout simplement gérer la masse de ses documents. Cette approche pragmatique cherche à voir comment des discours très généraux, voire emphatiques, une fois traduits en dispositifs et en outils, génèrent un certain nombre de questions quand on les aborde 
par le biais des pratiques quotidiennes. Il ne sera pas question de la construction organisationnelle en amont (c'est-à-dire faisant l'objet de décisions puis d'une mise en application et en règles) de ces formes communicationnelles mais de les considérer comme des cadres d'échanges (Delcambre 2009). Nous voulons traquer, sous l'activité des acteurs, leur activité d'information, sa place et la représentation qu'ils s'en font en reprenant leurs propres mots pour la décrire et non décrire les systèmes et ce qu'ils rendent possible.

De façon plus large, cela dessine les contours d'un monde informationnel, qui, à côté du monde réseau décrit par Boltanski (Boltanski 1999) aurait les caractéristiques suivantes : comme figure emblématique, celle du réservoir d'informations et de connaissances (local ou mondial) qui se décline en dispositifs, outils et systèmes d'information pour rendre disponible ce bien commun, selon certaines règles et comportements tant pour l'alimenter que pour en faire usage. C'est nous intéresser aux épreuves que constituent les interactions des individus avec ces systèmes lorsqu'ils veulent les plier à leurs attentes, que ce soit pour les exploiter, les construire, les faire vivre ou les amender.

4 Après avoir défini le cadre théorique de notre objet de recherche, nous illustrerons notre propos à l'aide d'entretiens de salariés avant de conclure sur plusieurs types de tensions et de paradoxes.

\section{Pratiques professionnelles et interactions}

5 Cette approche de l'activité info-communicationnelle nichée au cœur du travail mobilise et articule plusieurs éléments servant de points d'appui méthodologiques.

\section{Partir de l'activité de travail pour positionner l'information}

6 Sans remonter jusqu'à la théorie de l'activité et de l'action située dont nous nous inspirons, nous nous intéressons au travail sous l'angle de sa composante langagière (Borzeix 2005, Pène 2008, Delcambre 2009), particulièrement ici sous ses formes écrites; à côté de gestes concrets et, entrelacée avec eux, il y a toute une activité de mise en mots pour dire ce qu'on fait, pour orienter son action, pour se coordonner avec les autres, ces énoncés pouvant rester oraux ou être mis sur supports; ceux-ci peuvent alors circuler entre les personnes et alimenter des réservoirs d'information. C'est placer l'information au cœur de l'activité comme une ressource exploitée dans le cours de la tâche, en fonction de la place qu'occupe l'individu dans un processus et de son rôle, qui peut différer selon la situation. Il est acteur parce que réalisant de nombreux actes de nature différente, bénéficiant d'une certaine marge d'autonomie, de prise de décision et de responsabilité quant aux résultats de son travail et des moyens pris pour y parvenir. Il fait également preuve de réflexivité pour se représenter son activité et la mettre en mots, organisant ainsi une couche d'information qui la rend visible.

7 A cette première forme de capacité énonciative propre au travail s'ajoute celle de nommer les objets informationnels mobilisés. En faisant parler des techniciens sur les écrits qu'ils produisent ou utilisent Rouard et Moatty [2007] ont montré l'extrême variété des dénominations auxquelles sont associés des modes d'usage et d'utilité.

8 Nous sommes aussi au cœur des écrits de travail [Delcambre 2009] qui soulignent la relation entre information et action : agir sur ordre, selon des procédures, produire des 
textes pour soi-même ou pour d'autres, peut constituer non seulement le résultat de son activité (un chargé d'études, un chercheur), mais aussi relever d'une prescription pour justifier et rendre compte de son action. Les études sur la place du document dans ces échanges montrent combien sa circulation engage plusieurs niveaux de collectifs [Peyrelong, Guyot 2007] dans une géographie de l'information où se confirme que plus on s'éloigne de l'origine de l'énonciation, plus il importe de travailler à le rendre intelligible pour d'autres plus lointains [Thévenot 2006], travail d'éditorialisation [Guyot 2006] qui n'est pas toujours perçu comme faisant partie du travail de l'acteur. Que ces écrits soient maintenant déposés sur des plates-formes collectives rend encore plus pertinent de les considérer comme des surfaces d'inscription et de médiation, dotées de caractéristiques techniques, énonciatives [Souchier, Jeanneret 2003].

9 Se pencher ainsi sur l'acteur mobilisant ou gérant de l'information, laisse entrevoir une activité singulière, souvent totalement invisible car non prise en compte dans l'évaluation de son travail. Il y endosse de nombreux rôles, de chercheur / utilisateur / lecteur, de rédacteur / annoteur / valideur c'est-à-dire intervenant comme producteur d'énoncés, de gestionnaire (de documents et d'outils d'information), ou encore de partenaire (au sein ou hors de son collectif de travail). Chacun demande des actions et des compétences particulières, dans la mesure où il lui faut sans cesse repérer les différents dispositifs, la place que lui-même y occupe ainsi que les comportements / manipulations adaptés pour les faire concorder avec ses besoins. C'est ainsi que l'acteur agence autour de lui ces objets informationnels, en prenant soin d'identifier la façon dont chacun d'eux l'enrôle : comme destinataire d'un bulletin de salaire, émetteur d'un mél, gestionnaire de son propre système d'information, utilisateur d'une unité documentaire, producteur d'un avis... tout cela dans des degrés de variation propres à la tâche en cours.

\section{Objets circulant donnant à voir des dispositifs et systèmes informationnels}

Le poste de travail aujourd'hui s'avère extrêmement «équipé», voire encombré, d'objets, de technologies, d'outils, de dispositifs informationnels [Cotte 2008, Bailly 2002]. Nous prendrons particulièrement appui sur les documents parce que ce sont de bons observables, indicateurs des dispositifs et systèmes d'information: sites Internet, Intranets, books de procédures, bases mél, statistiques, bases comptes-rendus [Guyot 2006b].

11 Car ces objets-ressources font partie d'un système dans lesquels ils sont enrôlés de façon spécifique. Ils circulent à travers un système d'acteurs, ceux qui les produisent / énoncent / conçoivent et ceux qui les lisent, utilisent ou exploitent. Chacun est déterminé par des conditions de production (qui écrit, dans quelle position, pour quelle situation, qui définit le statut), par des caractéristiques d'accessibilité (faciles, directes ou médiatisées), d'utilisation (liées au statut, au dispositif, au langage d'interrogation...) ou de circulation entre personnes ou collectifs (en interne, entre gens du même métier, confidentialité...). Les règles ou normes (ou leur absence) du système organisationnel en régissent la production, la circulation et l'usage et un dispositif fait tenir ensemble et stabiliser ces objets munis de leurs règles à l'intérieur d'un cadre de fonctionnement, de circulation et d'utilisation. Ainsi, le simple fait d'être salarié fait automatiquement émarger à un dispositif d'information aux règles relativement stables (il reçoit son bulletin de salaire, pose une demande de congé selon un format pré-défini). Quant au 
service Ressources Humaines il gère les données nécessaires à son activité, ses propres procédures, fiches de poste, réglementation, cet ensemble pouvant être soutenu par des bases de données.

12 Un SI regroupe donc une variété de types d'information, un dispositif matériel pour y accéder (une personne difficilement joignable, une base de données sur l'intranet, une rubrique sur un site familier) et ne se réduit jamais au système informatique. Il se donne à voir à travers l'action des acteurs aux positions mouvantes. Il conviendrait de parler de système informationnel pour rendre compte de cette dynamique d'échanges d'informations et de documents entre acteurs selon des modalités parfois rigidifiées, parfois très souples, dépendant de la problématique suivie par l'acteur dans le cours de son travail : se mettre en relation avec un collègue, capter, sauvegarder des données, intégrer une statistique dans son rapport, se tenir au courant.

13 La présence croissante de réservoirs d'information hétérogènes ayant leur propre mode de gestion et d'accès peut s'avérer une source de difficultés pour l'individu, en termes d'accessibilité technique, de manipulation ou de démarche cognitive. Car outre les dispositifs /modalités pour accéder à l'information, chaque outil façonne une certaine attente de comportement qui exige de la personne une connaissance de ses codes techniques, sémiotiques, sémantiques et organisationnels. Car chacun d'eux assigne un rôle à l'individu qui y entre, et ces rôles sont interchangeables : il est destinataire de la lettre de l'entreprise, contributeur en rédigeant un article, gestionnaire en la classant dans son propre système, ou utilisateur lorsqu'il tente de la retrouver sur le site ${ }^{1} .$.

\section{Dire et interpréter}

14 Nous nous situons donc au plus près des interactions ordinaires lorsqu'elles mobilisent un objet informationnel. L'observation pointe un double travail, d'interprétation et de formulation. D'interprétation, pour identifier et décoder les différents systèmes d'écriture, d'organisation d'énoncés susceptibles d'être utilisés. D'énonciation, ensuite; dès lors qu'il cherche à pénétrer dans cet univers de mots et de textes (Delcambre 2009), les siens ou ceux des autres, l'acteur s'engage dans une action de formulation de sa propre intention, de sa démarche par rapport à la source, pendant la consultation ellemême, selon des formules et codes correspondant aux multiples contraintes techniques d'ordre logiciel, institutionnel, censées ouvrir des possibilités mais pouvant aussi agir comme des contraintes qu'il faut alors surmonter.

15 Notre définition de l'information rejoint alors celle des économistes, comme facteur de réduction d'incertitude ou de résolution de problème; de façon plus prosaïque, nous dirions, ce quelque chose qui manque à une personne pour avancer dans son travail, pour en formuler les résultats, pour entretenir ses compétences (Guyot, 2006).

16 Attachons-nous alors à ces opérations d'ajustement, de mise en correspondance, de la part d'un acteur naviguant dans un environnement complexe de codes, de dispositifs et d'outils, y sélectionnant ce qui lui convient. Il rencontre, voire se heurte, à des règles d'usage plus ou moins explicites et à des objets dont la stabilité n'est jamais acquise : les versions changent, les pages ou rubriques Internet sont fréquemment modifiées, au prétexte d'en améliorer l'ergonomie. Cela tend à déstabiliser la représentation que s'en fait l'acteur, l'incitant à mettre en place de nouvelles attitudes et à réaménager ses routines et chemins antérieurs (Alava 1996). 


\section{Les mots des acteurs}

17 Nous nous sommes appuyés sur la conscience discursive de chacun en interrogeant une cinquantaine d'acteurs pendant une heure sur leur activité de travail pour y déceler sa dynamique informationnelle. Les questions posées concernaient leur activité, leurs interlocuteurs habituels (réseau de travail) ainsi que les ressources mobilisées pour la réaliser. S'y lisent les composantes de l'activité d'information et ce que nous appelons leur système d'information personnel, constitué du réseau relationnel, des sources d'information et des modes de leur gestion documentaire [Guyot, 2006]. Il leur était également demandé de réaliser une recherche d'information sur une source couramment utilisée puis de présenter à l'enquêteur l'organisation de leurs dossiers et de leur bureau électronique qui condensent et résument l'activité et montrent la façon dont ils organisent leurs tâches par le simple fait de nommer fichiers et dossiers. Les personnes interviewées appartenaient au secteur tertiaire, assurant des fonctions aussi variées que chargés de mission, d'études ou de clientèle, assistants, responsables de service (juridique, économique, ressources humaines ou communication) ${ }^{2}$. Nous avons simplement ici regroupé sous quelques rubriques les mots mêmes des acteurs.

\section{Une activité d'information multiforme}

Décrire son activité est a priori une opération difficile car elle est perçue comme chaotique et non linéaire : «le problème, c'est qu'on fait tellement de choses »; elle se reproduit rarement à l'identique, fragmentée en tâches secondaires et souvent interrompue par des imprévus; "on est toujours dérangés ", «il y a tout ce qu'on ne maitrise pas », « tout le reste ».

19 Ce sont des verbes d'action qui la décrivent. Ainsi un responsable de service en ingénierie de formation identifie ainsi : «je rencontre (le client), j'analyse, je transcris, je rédige (une proposition), je fais valider, je demande, je choisis, je transmets (à un formateur, au client), je crée (un formulaire d'évaluation), je me déplace (pour animer les bilans de fin de stage)» chaque action étant générée et générant des documents (compte-rendu, cahier des charges, document comptable, fiche logistique, formulaire, note). En tant que responsable du service, il « transmet » et « fait remonter l'information » à ses collègues et à sa hiérarchie, « relaie l'information nationale, rédige des bilans d'activité, alimente un outil de suivi, recense les actions menées" pour conclure "c'est beaucoup de rédactionnel et de réunions ".

20 Une restauratrice d'objets muséaux dit: «le conservateur me transmets le dossier et l'objet; je constate l'état » (qui donne lieu à une étude technique) puis « tout au long de la restauration je la documente en prenant des photos des différentes étapes, avec des étiquettes et des numéros d'inventaire ; on explique les produits qu'on utilise, on fait des préconisations, on garde toute l'information relative aux objets ", on y associe des dossiers de fouille. Toutes ces traces seront ensuite classées dans le « dossier d'œuvre » au centre de documentation, et gardées sur son propre ordinateur.

Un responsable juridique prend l'exemple d'un appel d'offres : «le service me donne son cahier des charges, je le valide juridiquement, rajoute des clauses qui sont absentes et après je rebascule au service concerné ». Par delà ces transferts entre acteurs participant au même processus, il y a aussi toute une activité de recherche d'informations. 


\section{S'informer} "preuve» «je sors la feuille pour avoir une trace en cas de contestation». Il sert également de moyen de stockage «j'essaie de conserver la documentation que je reçois ; ça peut servir pour faire des statistiques ", dans la dynamique papier / électronique, le papier sert de pense-bête ou est utilisé pour « lire plus tard » comme un arrêt dans le flux électronique continu qui demande à être géré : mise en dossiers, repères de couleurs, alertes... fallait téléphoner partout, choper le collègue en Angleterre, là c'est très facile ", ce recours aux bases d'information n'est pas toujours aisé : «les informations se perdent dans la masse »; « je n'utilise pas internet comme base, je n'ai pas le temps ; si t’a pas déjà une idée tu vas te perdre dans les méandres; si tu as une approche probabiliste, si tu sais 
estimer le temps que tu y passeras et ce que ça va te rapporter, ok, tu fais un arbitrage » «je sais ce que je voulais, si tu ne sais pas... »; «Je n'utilise pas les bases documentaires, j'utilise les documentalistes, je suis une fainéante »; « Je vais y aller, mais je ne sais plus comment j'ai fait, pourquoi je ne retrouve pas, il y avait un lien...»

Sous ces remarques se profile une représentation de ce qui est possible d'obtenir et d'une stratégie de sélection. Face à la prolifération d'outils, l'acteur met en place des routines parce que «si on ne va pas chercher l'information, personne ne nous la donne » dans une dynamique volontaire ou passive : «je préfère recevoir un document papier qu'aller sur le site », je n'y vais pas spontanément»; "quand on t'apporte l'information, c'est vraiment le meilleur moyen ", " je les reçois sans avoir jamais rien demandé, on a dû me mettre dans la boucle». Cette activité de recherche demande «beaucoup de persévérance », beaucoup de clics et de détours »; «il faut se donner du temps [pour] agrandir mes ressources d'information »; «c'est des méthodes qu'il faut acquérir et je n'ai pas forcément le réflexe ».

\section{Les limites du partage}

Les organisations se préoccupent actuellement de ce que nous pourrions intituler un déversement des systèmes d'information personnels (SIP) dans un espace collectif. A une organisation individuelle de l'information s'ajoutent celles des outils ou systèmes collectifs développés par la structure et supposés mutualiser tant l'information détenue par chacun que celle qui est considérée comme utile à tous. Cela incite à réfléchir aux rapports de co-existence, concurrence ou complémentarité qui s'établissent entre ces systèmes, ce qui pourrait suggérer quelques limites au vieux rêve d'une mise en commun totale.

\section{Des systèmes d'information très personnels (SIP) parfois partageables}

De l'avis des personnes interrogées, la gestion personnelle de l'information s'apparente au travail administratif : remplir des fiches, conserver ses papiers, archiver les courriels, classer ses dossiers: «j'ai un classement par année, par affaire, je m'y retrouve, je me débrouille; «je les mets, mais après je les exploite pas comme je devrais »; «j'ai deux classements, par pays et par organisme, je mets toujours un numéro sur mes notes ; c'est le plan de classement du Ministère [...] si on va sur l'espace commun c'est incompréhensible, il y a aussi bien des dossiers du personnel que des notes, c'est d'une grande confusion, on ne trouve rien; donc nous on a fait un truc et là on trouve notre classement. Tout le monde $\mathrm{y}$ a accès ; mais j'ai aussi un dossier à moi, quand les choses ne sont pas finalisées, tout est là, par ordre alphabétique avec des thématiques [...] ça se fait petit à petit ; sur les méls, j'ai une organisation par pays et projet... mais je n'y arrive pas, je ne sais pas quoi en faire, tiens, ça ne devrait pas être là, donc je le fais ponctuellement, mais quand je fais des recherches je ne les retrouve pas automatiquement. Si déjà je prenais le temps de suivre ce que j'ai décrété, ce serait pas mal, mais je ne prends pas ce temps ».

Mais ce système "artisanal » apparemment efficace parce qu'en phase avec l'activité, révèle ses limites dans le partage. Il peut être prêté par son propriétaire qui en autorise l'accès et décide de le partager : « j'ai envoyé mon rapport car je savais qu'ils travaillaient sur ces sujets et ça pouvait les intéresser ». Mais «si les gens savent où se trouvent les 
documents, comment ils sont classés, quand on est absent je ne sais pas comment les trouver. Pour tout ce qui est numérique, ils n'ont pas accès à l'information, les gens ne se permettent pas déjà d'ouvrir l'ordinateur d'une personne absente, et en plus chacun a son propre classement, donc c'est pratiquement impossible de s'y retrouver. Je serais incapable de le trouver par moi-même ". Si le classement de l'un ne correspond pas au classement de l'autre, où et comment trouver le bon arrangement, c'est-à-dire le dénominateur commun?

\section{Connecter systèmes personnels et systèmes collectifs}

31 Car cette organisation engendre une redondance chronophage et coûteuse, de microsystèmes hétérogènes non inter-opérables qui concurrencent ou complètent des dispositifs collectifs pour mutualiser l'information et réduire ce travail individuel de gestion. Les outils quotidiens d'un service se retrouvent sur des espaces dédiés, et sur le serveur commun cohabitent désormais répertoires personnels et dossiers partagés : «on met nos trucs de travail »; «j'ai sur mon disque dur un tas de trucs; j'avais commencé à organiser tous les brouillons, et après je vais les mettre (une fois finalisés) sur le « $\mathrm{S}$ " (serveur commun), c'est totalement idiot, ça ne marche pas, cela fait perdre du temps [...] à un moment donné je dis je le garde et je le bascule sur le $\mathrm{S}$ ». Tout se joue sur la cohérence, l'intelligibilité des informations ainsi postées, et l'on voit un fort décalage entre les objectifs assignés à de tels systèmes et la réalité vécue par les acteurs.

Aux notions d'information contextuelle ou opérationnelle qui signalent le type d'usage, il conviendrait d'ajouter celles d'outil de travail et d'outil d'information. Car un système d'information peut être tellement intégré à la tâche qu'il en devient une partie inhérente (cas d'un processus commercial qui regroupe l'état des stocks et des commandes, une base retours-clients, un contrat-type, un formulaire ou les procédures de travail faisant obligation d'utiliser un outil adapté à chaque étape afin de savoir immédiatement où on en est, ce que font les autres, vérifier les délais de livraison...). L'usage du système est incontournable car l'information suit le processus même, elle en signale les phases. L'outil d'information relève de l'éditorial et du documentaire, avec une stratégie de ciblage des destinataires ; l'information est plus éloignée du quotidien, on peut avancer sans elle. Elle constitue une ressource éventuelle pour vérifier un point juridique, une procédure, aller chercher une fiche technique constructeur ou se tenir au courant des activités et des projets de l'organisme,.

Décrire un système suppose de spécifier ceux qu'il concerne, ce qu'on y trouve, ce qu'on peut en faire, son aire de validité (locale, l'ensemble de la structure). Si un Intranet constitue toujours un outil d'information (institutionnel, documentaire) il est parfois aussi un outil de travail, ce qui explique que son appréhension soit complexe : « je n'ai pas été assez curieux pour voir ce qu'il y a dedans, mais on ne m'a pas donné envie de voir dedans », « on doit cliquer partout, on ne sait pas où regarder [...] « je regarde l'annuaire, l'organigramme, tout ce qui est suivi des facture, j'utilise aussi tous les documents type ; je clique un peu partout jusqu'à ce que je trouve "; « sur la base, tu tapes son nom... pas de réponse, c'est un de nos experts favoris, donc tu dis peut-être y a $t$ il un de ces vieux trucs de mettre en majuscule... pas de réponse, oublions le formulaire, laisses tomber... je ne cherche que dans le texte entier»; «il faut que je m'amuse à aller chercher toute la liste pour trouver ». « c'est archi-bourré » " voyez, c'est incompréhensible, on ne sait pas à quoi cela correspond ». " c'est plus des informations générales, ce n'est pas du tout un intranet comme cela pourrait l'être, on a vraiment tout ce qu'il faut pour mettre en place 
mais après il y a la volonté ». Est ainsi reconnu le travail préliminaire de construction qui s'appuie sur une analyse des processus et des ressources afférentes.

Les interviewés expriment des attentes fortes pour un système unique, fiable, toujours disponible, à jour, organisé selon leurs propres critères, répondant à toutes leurs questions et donnant des informations sur chaque service, car : « ce serait bien de savoir ce que font les autres ", « si on mettait ensemble nos dossiers ", « si je pouvais le trouver quand elle n'est pas là », « en faire profiter l'ensemble des services et ensuite faire part de leur commentaire, cela permettrait de se tenir informé, de faire une bibliothèque virtuelle "; " pas vraiment de méthode d'archivage, on a beaucoup d'envies »; « on reste chacun de notre côté et comme on n'a pas le temps. Cette base de données (commune) apparait plus comme une contrainte... en disant que ça ne sert à rien... je l'utilise comme une chambre d'enregistrement"; "permettre aux autres services de profiter de nos compétences, après ils en font ce qu'il veulent $»$.

Ces phrases donnent une idée des cloisonnements organisationnels venant entraver la fluidité du travail et générer une méconnaissance réciproque : «qui fait quoi »; «avant ils faisaient circuler mais maintenant ils n'ont plus le temps; c'est vrai qu'en cas de besoin je vais voir $\mathrm{X}$ qui m'apporte certains éclaircissements mais généralement je me débrouille de mon côté ». D'où des déplacements, des initiatives multiples pour obtenir une information, celle que possède une personne ou celle qui se trouve dans un réservoir d'information.

Mais les systèmes prescripteurs, mis en place par l'organisme, ne fonctionnent pas toujours bien : «quand les gens renseignent les tableaux, c'est quand ça fait partie des procédures »; «on était censé mettre tous nos documents (de travail, un courrier envoyé) ; personne ne l'a utilisé, je pense que personne n'avait envie de le faire, pourtant l'outil est installé sur nos ordinateurs ». Mais parfois le système s'est progressivement ajusté pour résoudre un problème rencontré "on avait mis en place un circuit de validation. Ça n'a jamais vraiment bien fonctionné, quand on a affaire à des populations si différentes, chaque service a une certaine manière de travailler, de penser, donc il faut essayer de s'adapter à leurs méthodes de travail; il y a eu peut-être un manque à être pro-actif, c'est-à-dire à mettre en place un peu par la force ».

\section{Une affaire d'attitude}

37 Si les personnes s'accordent sur le principe du partage, elles constatent qu'il introduit un changement dans les façons de travailler : « cela va être difficile de le faire admettre aux autres... on essaie d'ouvrir la base de données à l'ensemble des services concernés pour qu'ils puissent faire part de leurs commentaires pour avoir un circuit de validation un peu plus rapide ... c'est un outil d'enregistrement alors que ça pourrait être un outil de circulation plus fluide »; «j'avais créé un espace de valorisation de la recherche [...] pour permettre aux praticiens, depuis leur place, de faire résonner [...] et de faire des groupes qui réagissent [...] mais cela n'a pas marché, je renonce à faire écrire les praticiens, c'est trop compliqué, ils ont peur d'écrire et / ou de ne pas être au niveau, ou alors ils sont tellement submergés qu'ils n'ont pas le temps ». Tout changement dans la configuration technique ou organisationnelle impacte les acteurs et leurs façons de travailler.

Cela entraîne alors potentiellement une tension entre des attentes (personnelles, institutionnelles) et le mode de fonctionnement des personnes. Cela pose aussi la question de la légitimité à proposer des solutions et les faire accepter par les collègues. Si 
tous ont conscience d'améliorations possibles, ils ne savent pas trop qui serait légitime pour offrir un système commun qui tienne compte des intérêts de chacun.

\section{Conclusion} qui le relie aux autres ainsi que ses moyens informationnels, suscite plusieurs réflexions. La première montre une fois encore combien l'acte de travailler demande une intense activité cognitive, singulière et récurrente, d'information aux fortes composantes communicationnelles, qui s'accompagne d'actes concrets devenus complexes dès lors qu'il faut jongler avec de nombreux outils et dispositifs, penser aux autres en même temps qu'à soi-même, se fixer ou respecter des règles collective, ou encore comprendre la logique de l'autre, intercompréhension pouvant parfois se révéler difficile.

La seconde souligne plusieurs types de tensions: entre l'activité de travail et l'activité d'information et, à l'intérieur de cette dernière, une tension entre le fait d'alimenter un réservoir, commun ou personnel, et le fait de les exploiter. Sur le plan organisationnel, les acteurs s'expriment longuement, et volontiers, sur cette part de leur travail, aussi bien celle qui est requise lorsqu'ils participent à un outil commun, que celle qui fait d'eux un utilisateur. Il y a là des degrés de proximité, d'affinité, d'engagement informationnel qui restent à approfondir, dans un mouvement permanent pour trouver le rapport qui convient entre investissement et retour sur investissement, tant individuel qu'organisationnel.

Ce travail artisanal, imputé à une formation insuffisante car " générique ", sans rapport direct avec les situations personnelles, nécessite du temps pris sur le travail productif afin de banaliser, "routiniser" de nouvelles pratiques avec des outils fréquemment modifiés. Cela raidit davantage le rapport entre l'exigence de résultats et l'exigence croissante de mettre l'activité en mots et en données, traçabilité de l'action propre à une bureaucratisation désormais attendue de chaque acteur, tout comme de sa « disponibilité » (Pène 2008).

Cette enquête précise quelques contours d'un bien commun informationnel dont il est rêvé qu'il circule sans entraves, universel et libre de droits. Nous avons insisté sur l'hétérogénéité des épreuves visant à faire concorder l'information aux besoins et liés aux situations tout en créant des outils communs. Cela explique une tension constitutive entre des logiques personnelles pour tordre une information par l'interprétation et une logique collective pour œuvrer à ce bien commun. Cette tension nous paraît faire partie de la dynamique informationnelle qui relie personnes et systèmes. Elle existe parce qu'on ne gère pas pareillement une information devenue sienne une fois qu'on l'a adaptée à ses besoins (ce qui correspond à une logique personnelle et pragmatique) et celle qu'on poste sur un système collectif : produire une information pour d'autres renvoie à une logique de service dont la principale forme est ici éditoriale.

Cela expliquerait l'échec de certaines initiatives individuelles pour créer un outil au service d'un collectif. Par absence de réflexion sur le dispositif d'alimentation, de mise à jour et d'usage ou par déficit de légitimité organisationnelle des initiateurs, ou encore par un statut relativement informel ou une carence de confiance et de motivation des autres participants. De surcroit, une juxtaposition d'informations individuellement déposées ne garantit pas automatiquement un système commun et seule une attention éditoriale rend possible son appropriation, voire son annexion aux systèmes personnels. Cette logique 
éditoriale est associée à une logique organisationnelle dont le modèle serait la négociation, même si elles apparaissant comme un investissement et une charge de travail consistant. Cela peut expliquer que cette économie contributive en cours d'instrumentalisation n'en soit qu'à ses premiers pas.

Il n'est pas certain qu'un individu immergé dans son travail puisse toujours mener à bien cette activité éditoriale qui consiste à passer d'un à moi ou un à eux pour aboutir à un à nous. Si beaucoup d'entre eux en soulignent la nécessité, ils la perçoivent aussi comme potentiellement contradictoire avec la réalisation de leur tâche, encore que celle de s'informer leur parait moins contraignante que celle qui consiste à informer les autres. Alors, qui est porteur et qui est responsable de ce bien commun? en faut-il ? Peut-être atteignons-nous ici les limites du partage, souhaité à la fois par les organisations et les personnes mais qui se révèle fort complexe à mettre en œuvre. Ce n'est pas seulement par manque de réflexion, c'est aussi parce que cela touche de près chaque acteur dans son rapport au monde et aux autres.

On voit bien que cette culture de l'information dépasse largement les seuls aspects manipulatoires ou méthodologiques et inclut les façons dont les personnes se représentent les ressources informationnelles. C'est une culture d'appariement, tant des mots aux documents que des besoins aux documents, ou encore que des personnes entre elles. Elle garde une composante communicationnelle largement informelle qui déborde des outils mis en place, et dont il n'est pas certain, ni nécessairement opportun, qu'elle se laisse totalement encadrer. Elle est en tout cas en passe d'être identifiée, mise en mots et en rapport avec des fonctionnements organisationnels dont on peut voir qu'ils oscillent entre imposition et laisser-faire, qui jouent sur la bonne volonté ou la pression. Une affaire à suivre, certainement.

\section{BIBLIOGRAPHIE}

Alava, S., (1996), « Autoroutes de l'information et apprentissages documentaires » documentaliste - sciences de l'information, vol 33, $\mathrm{n}^{\circ} 3$.

Bailly, F., Blanc, M., Dezalay, T., Peyrard, C., (2002), Pratiques professionnelles et usage des écrits électroniques, l'Harmattan.

Boltanski, L., Chiappello, E., (1999), le nouvel esprit du capitalisme, Gallimard.

Borzeix, A., Fraenkel, B., dir., (2005), Langage et travail. Communication, cognition, action, Paris, CNRS.

Bouillon, JL., (2008), « l'impensé communicationnel dans la coordination des activités socioéconomiques ", sciences de la société $\mathrm{n}^{\circ} 74$.

Cotte, D., (2007), espace de travail et logique documentaire, études de communication $n^{\circ} 30$.

Delcambre, P., (2009), « un état des recherches sur les « communications organisationnelles »en France : l'âge des transmissions ", Sciences de la société, $\mathrm{n}^{\circ} 74$.

Guyot, B., (2006a), Dynamiques informationnelles dans les organisations, Hermès-sciences. 
Guyot, B., Peyrelong, MF., (2006b) « le document dans une perspective organisationnelle : un objet comme un autre? ", sciences de la société $\mathrm{n}^{\circ} 68$

Lépine, V., (2002), « Une entreprise sidérurgique aux prises avec le groupware : apports d'une étude empirique, " revue les enjeux, Gressec http://w3.u-grenoble3.fr/les_enjeux/2002/Lepine/ home.html\#debut

Linhart, D., (2009), Travailler sans les autres? Seuil.

Moatty, F., Rouard, F., Teiger, C., (2007), « Lectures pour soi, lectures invisibles ? Une cartographie des lectures au début de l'internet », in A.-F. de Saint Laurent-Kogan, J.-L. Metzger, JL., (dir.), Où va le travail à l'ère du numérique ?, Les Presses ParisTech, coll. « Sciences sociales ».

Pène, S., Borzeix, A., Fraenkel, B., dir., (2001), Le langage dans les organisations. Une nouvelle donne , Paris, L'Harmattan.

Pène, S., (2005), « La Vie des hommes infâmes dans la société de disponibilité », Études de communication $\mathrm{n}^{\circ} 28$.

Souchier, E., Jeanneret, Y., Le Marec, J., (2003) Lire, écrire, récrire: objets, signes et pratiques des médias informatisés, BPI.

Thévenot, L., (2006), L'action au pluriel : sociologie des régimes d'engagement, La découverte.

\section{NOTES}

1. De nombreux travaux s'attachent à étudier les systèmes d'information du point de vue organisationnel , notamment JL Bouillon, V Lépine, M Durampart...

2. Le but n'était pas d'établir des comparaisons ni des typologies, mais bien de comprendre le point de vue de la personne. Un certain nombre d'entretiens ont été conduits par nos étudiants à partir d'un guide d'entretien commun.

\section{RÉSUMÉS}

Faire parler les acteurs de leur activité d'information donne à voir l'hétérogénéité des systèmes et outils qui entourent leur poste de travail et des façons de les mobiliser. L'article s'appuie sur l'expression même des acteurs, sur leurs façons de nommer leur travail et les ressources propres à le faire avancer. Il montre l'intense dynamique qui se joue autour et avec des outils d'information, pour produire de l'information ou en échanger, ou encore pour participer à des dispositifs collaboratifs. Cela fait apparaître une tension entre le système personnel que développe chaque acteur et les dispositifs collectifs, notamment lorsqu'il est question de leur partage et de leur mutualisation.

Make persons speak of their information activity shows the heterogeneity of information systems and tools that surround their workplace and of ways of mobilizing it. The article is based on the expression of the actors themselves about their way to work and appoint their information resources. It shows an intense dynamic and various plays with information tools to produce or exchange information, or to participate in collaborative systems. This reveals a 
tension between the personal system that each person develops and collective information devices, especially when it comes to sharing and pooling them.

\section{INDEX}

Keywords : information activity, information system, collaborative device, information sharing, work, professional practice

Mots-clés : activité d'information, partage d'information, dispositifs collaboratifs, système d'information, travail, pratique professionnelle

\section{AUTEUR}

\section{BRIGITTE GUYOT}

Brigitte Guyot est maître de conférences en SIC au CNAM, membre de l'équipe DICEN (dispositifs d'information et de communication à l'ère numérique) mène des recherches en sociologie de l'information. Elle travaille plus particulièrement sur l'interconnexion des systèmes d'information en entreprise et sur les façons dont on travaille avec eux. Elle base son approche sur les documents qui circulent dans le cours de l'activité, pour y étudier les transformations organisationnelles en cours.

brigitte.guyot@cnam.fr 